\title{
ACESSO E ACESSIBILIDADE NA ATENÇÃO PRIMÁRIA À SAÚDE NO BRASIL
}

Ellen Christiane Corrêa Pinhol

Thais Amanda Nunes da Cunha ${ }^{1}$ Messias Lemos ${ }^{1}$

Glenda Roberta Oliveira Naiff Ferreira ${ }^{1}$ Luciano Garcia Lourenção

Helder Henrique Costa Pinheiro ${ }^{1}$ Eliã Pinheiro Botelho ${ }^{1}$

Carlos Leonardo Figueiredo Cunha ${ }^{1}$

\begin{abstract}
https://orcid.org/0000-0001-8491-3381 https://orcid.org/0000-0002-7590-0308 https://orcid.org/0000-0002-6267-599X https://orcid.org/0000-0002-8206-4950 https://orcid.org/0000-0002-1240-4702 https://orcid.org/0000-0001-5567-3550 https://orcid.org/0000-0002-9682-6530 https://orcid.org/0000-0002-1891-4201
\end{abstract}

Objetivo: Avaliar o acesso e a acessibilidade às Unidades Básicas de Saúde nas diferentes regiões do Brasil, caracterizando-os segundo os componentes organizacional e de infraestrutura. Método: Pesquisa de natureza avaliativa, através de um estudo transversal com abordagem quantitativa, fundamentado nos dados do terceiro ciclo do Programa Nacional de Melhoria do Acesso e da Qualidade da Atenção Básica. Resultados: A maioria das Unidades Básicas de Saúde $(84,4 \%)$ possuem horário fixo de funcionamento; as unidades da região Norte revelaram os horários mais variáveis (72,0\%); em média, 46,3\% das unidades mantém atividades no horário do almoço, sendo que regiões Centro-Oeste (24,2\%) e Sul (29,5\%) concentram os menores percentuais de unidades com funcionamento em horário de almoço; $87,1 \%$ apresentaram todos os ambientes com sinalização, facilitando acesso dos usuários; poucas unidades possuiam corrimão nos locais não nivelados (19,6\%) e piso tátil para acesso às dependências (24,1\%); 21,7\% apresenta estrutura divergente do estabelecido pelo Ministério da Saúde. Houve disparidades regionais nos achados encontrados em âmbito nacional quanto ao acesso e à acessibilidade. Conclusão: Na avaliação geral, o componente organizacional aponta conformidade com o preconizado pelo Ministério da Saúde e, no componente infraestrutura, a acessibilidade indicou percentuais que demandam avanços para melhoria do acesso.

Descritores: Acessibilidade aos Serviços de Saúde; Unidade Básica de Saúde; Atenção Primária à Saúde.

\section{ACCESS AND ACCESSIBILITY IN PRIMARY HEALTH CARE IN BRAZIL}

Objectives: Evaluate access and accessibility to Basic Health Units in different regions of Brazil, characterizing them according to organizational and infrastructure components. Method: Evaluative Research through a cross-sectional study with a quantitative approach, based on data from the third cycle of the National Program for Improving Access and Quality in Primary Care Results: Most Basic Health Units (84.4\%) have fixed opening hours; units of the North region revealed the most variable hours (72.0\%); on average, $46.3 \%$ of the units maintain activities at lunchtime, with the Midwest (24.2\%) and South (29.5\%) regions having lowest percentage of units operating during lunch hours; $87.1 \%$ presented all environments with signage, facilitating access of users; few units had handrails in non-level locations (19.6\%) and tactile flooring for access to premises (24.1\%); $21.7 \%$ presents a structure that differs from that established by the Ministry of Health. There were regional disparities in the findings found nationwide regarding access and accessibility. Conclusion: In the overall assessment, the organizational component points to compliance with the recommended by the Ministry of Health, and the infrastructure component, accessibility indicated percentages that require advances to improve access.

Descriptors: Accessibility to Health Services; Health Centers; Primary Health Care.

\section{ACCESO Y ACCESIBILIDAD EN LAATENCIÓN PRIMARIA DE SALUD EN BRASIL}

Objectivos: Evaluar el acceso y la accesibilidad a las Unidades Básicas de Salud en diferentes regiones de Brasil, multiplasándolas de acuerdo con los componentes organizativos y de infraestructura. Metodo: Investigación de carácter evaluativo, a través de un estudio transversal con un enfoque cuantitativo, basado en datos del tercer ciclo del Programa Nacional para Mejorar el Acceso y la Calidad en Atención Primaria. Resultados: Más Unidades Básicas de Salud (84,4\%) tienen horarios de apertura fijos; las unidades en la región norte revelaron las horas más variables (72.0\%); en promedio, $46.3 \%$ de las unidades mantienen actividades durante las horas de almuerzo, con las regiones del Medio Oeste (24.2\%) y Sur (29.5\%) que tienen el porcentaje más bajo de unidades operando durante las horas de almuerzo; $87,1 \%$ presentó todos los entornos con señalización, facilitando el acceso de los usuarios; pocas unidades tenían pasamanos en ubicaciones no niveladas (19.6\%) y pisos táctiles para acceso a las instalaciones (24.1\%); 21,7\% presenta una estructura que difiere de la establecida por el Ministerio de Salud. Hubo disparidades regionales en los resultados encontrados en todo el país con respecto al acceso y la accesibilidad. Conclusión: En la evaluación general, el componente organizacional apunta al cumplimiento del componente recomendado por el Ministerio de Salud y del componente de infraestructura, la accesibilidad indicó porcentajes que requieren avances para mejorar el acceso.

Descriptores: Accesibilidad a los servicios de salud; centros de salud; atención primaria de salud. 


\section{INTRODUÇÃO}

Os avanços na Atenção Primária à Saúde (APS) no Brasil são inegáveis, com mais de 43 mil equipes da Estratégia Saúde da Família (ESF) atuando em todo o país. Todavia, a precariedade da estrutura física das Unidades Básicas de Saúde (UBS) ainda se apresenta como um importante desafio(1). Análises de programas e serviços de saúde, no contexto da avaliação do acesso e acessibilidade da estrutura das UBS demostraram que a estrutura pode interferir na organização dos serviços, na qualidade da assistência prestada, além de gerar insatisfação e limitações nos profissionais da saúde e usuários ${ }^{(2-5)}$.

A estrutura faz parte da tríade Donabediana de avaliação da qualidade dos serviços de saúde, que envolve a "estrutura", "processo" e "resultados". Para Donabedian"(6), a estrutura refere-se aos instrumentos, recursos materiais e humanos, e contexto físico e organizacional dos serviços de saúde.

O Programa de Melhoria do Acesso e da Qualidade da Atenção Básica (PMAQ-AB)(7) do Sistema Único de Saúde (SUS), é baseado na tríade Donabediana, sendo considerado como a principal estratégia de avaliação da Atenção Básica à Saúde (ABS) no Brasil e objetiva induzir a ampliação do acesso e melhoria da qualidade desta.

Posto isso, pesquisas com pessoas com deficiência que buscam os serviços ofertados pelo SUS elucidam que a garantia da acessibilidade física e a comunicativa, são consideradas referenciais da qualidade dos serviços, e expõem problemas no acesso por conta das limitações físicas ${ }^{(8-10)}$. Além destes, obstáculos para a marcação de consultas, horários de funcionamento e implantação não equânime das UBS resultam em dificuldade no acesso da população( ${ }^{(9,11-12)}$.

Para este estudo considerou-se acesso como a possibilidade de o usuário entrar no serviço de saúde, na busca pela solução de seus problemas e está relacionado com a localização geográfica da unidade de saúde, os dias em que o atendimento é ofertado, a disponibilidade de horários e a possibilidade de atendimento sem agendamento prévio ${ }^{13}$. Já a acessibilidade consiste no ajuste entre as características dos recursos de saúde e as da população, no processo de busca e obtenção de assistência à saúde, e permite identificar os fatores que facilitam ou obstaculizam a busca e obtenção desta assistência. Resulta de uma combinação de fatores de distintas dimensões, como de ordem geográfica, organizacional, sociocultural e econômica(14).

Ressalta-se também a relevância da análise socioespacial quanto ao acesso e à acessibilidade da população, pois existem desigualdades referentes aos indicadores de estrutura dos serviços primários entre as Unidades Federativas (UF) ${ }^{(15)}$.

Logo, reconhecendo a APS como a porta de entrada aos serviços de saúde do SUS, monitorar e avaliar seu desempe- nho é fundamental para a mensurar seu alcance e qualidade, de forma a proporcionar subsídios para a tomada de decisões, além de possibilitar a melhor visualização das diferenças socioespaciais quanto ao acesso aos serviços primários de atenção à saúde(16-17).

Assim, este estudo objetivou avaliar o acesso e a acessibilidade às UBS nas diferentes regiões do Brasil, caracterizando-os segundo os componentes organizacional e de infraestrutura.

\section{MÉTODO \\ Tipo de Estudo}

Pesquisa de natureza avaliativa, através de um estudo transversal com abordagem quantitativa, fundamentado nos dados do terceiro ciclo do Programa Nacional de Melhoria do Acesso e da Qualidade da Atenção Básica (PMAQ- AB), do Ministério da Saúde (MS). Especificamente, fez-se um recorte dos dados do Terceiro Ciclo da Avaliação Externa, que ocorreu durante os anos de 2017 e 2018, publicados em junho de 2019.

\section{Local do Estudo}

O estudo abrangeu todo o território nacional, utilizando a divisão político-administrativa das cinco regiões: Norte, Nordeste, Sudeste, Sul e Centro-Oeste. Embora a distribuição populacional, as condições geográficas, ambientais e socioeconômicas sejam distintas nas diferentes regiões, a estruturação das UBS deve seguir modelo preconizado pelo Ministério da Saúde, em termos de infraestrutura mínima, estabelecido pela Política Nacional da Atenção Básica (PNAB). ${ }^{(16)}$

\section{Participantes do Estudo}

Os dados do PMAQ-AB são coletados por um pesquisador, previamente capacitado, que se desloca até uma unidade previamente definida e agendada pela nucleadora da pesquisa a Secretaria Municipal da Saúde. Ao chegar à UBS um sujeito informante chave que atua nesta, passa a responder todas as perguntas do questionário online, que ao final, são validadas pelo pesquisador. Neste estudo, não foi pedido aos informantes-chaves que assinasse o Termo de Consentimento Livre e Esclarecido (TCLE) por se tratar de dados secundários do Sistema de Informação do Ministério da Saúde. Assim, os componentes a serem estudos, serão as UBS.

A população do estudo foi composta pelas 30.346 UBS distribuídas nos 5.324 municípios (95,6\% do total) brasileiros que aderiram ao PMAO-AB e passaram pelo Terceiro Ciclo da Avaliação Externa.

Para tanto, foram incluidas no estudo as UBS que atenderam o seguinte critério de inclusão: conter informações da avaliação externa nas categorias dos componentes organizacional e de infraestrutura. Foram excluídas as UBS que tinham 
horário de funcionamento inferior a quatro horas por dia; apresentaram como resultado "não respondeu ao questionário" ou tinham ausência de resultados nas variáveis.

\section{Coleta dos Dados}

Os dados foram coletados no mês de outubro de 2019, por meio do Portal da Secretaria de Atenção Primária à Saúde (SAPS) ${ }^{(18)}$, do Ministério da Saúde, no escopo "Ações, Programas e Estratégia" - PMAQ, 3으 Ciclo, pasta "Microdados da Avaliação Externa".

As variáveis utilizadas neste estudo correspondem ao "Módulo I" da Avaliação Externa do PMAQ-AB - Observação na UBS, e condizem com o espaço organizacional e estrutura física, com aspectos referentes ao acesso e à acessibilidade. Perguntas que não se aplicavam ao contexto da unidade foram excluídas.

Após a obtenção dos arquivos com os microdados fornecidos e estruturados em matrizes de dados no software Microsoft Excel@, foi realizada a depuração do banco e foram selecionadas seis variáveis (regiões brasileiras; horário fixo de funcionamento; funcionamento em horário de almoço; componentes de acessibilidade nas dependências; disponibilidade de sanitário com acessibilidade; condições de funcionamento), que foram reorganizadas para possibilitar análises mais apuradas. Posteriormente, foram aplicados os critérios de inclusão e exclusão.

\section{Procedimentos de análise e tratamento dos dados}

Os dados foram, então, categorizados por regiões bra-

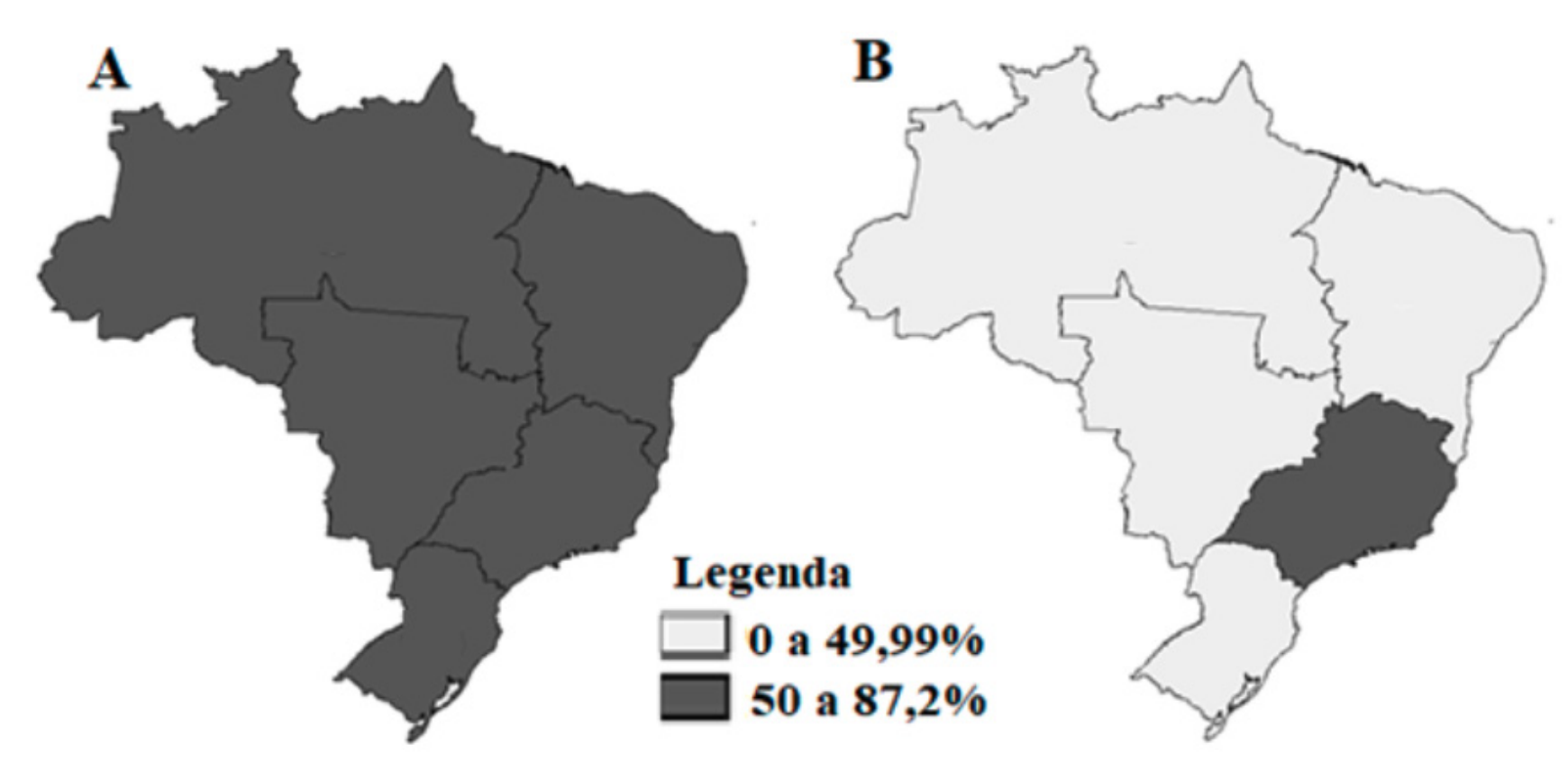

sileiras e analisados com o software IBM SPSS Statistics, versão 20.0, por meio de estatística descritiva, utilizando-se frequência absoluta e relativa, e apresentados em forma de tabelas e figuras.

\section{Procedimentos Éticos}

Por se tratar de dados obtidos de fontes secundárias, de domínio público, é dispensável a submissão deste projeto à apreciação do Comitê de Ética em Pesquisa (CEP), conforme estabelece o parágrafo único do Artigo 1으, da Resolução no 510 , de 7 de abril de 2016, do Conselho Nacional de Saúde (CNS).

\section{RESULTADOS}

\section{Componente Organizacional: Horário de Funcionamento}

Conforme observado na Figura 1, a maioria das UBS $(84,4 \%)$ possuem horário fixo de funcionamento (Imagem A), sendo que as unidades do Nordeste foram as que mais indicaram horário fixo de funcionamento (87,2\%), seguido da região Sul (85,9\%), Sudeste (83,3\%) e Centro-Oeste (83,2\%). De forma contrária, as UBS da região Norte revelaram variação nos horários de funcionamento (72\%).

Somente $46,3 \%$ das UBS mantém suas atividades no horário do almoço (Imagem B). A região Sudeste indicou o maior número de UBS funcionantes nesse horário (72,7\%), seguido pela região Norte (43,2\%) e Nordeste (38,3\%). As regiões Centro-Oeste $(24,2 \%)$ e Sul $(29,5 \%)$ concentram menor percentual de unidades que mantem atividades em horário de almoço.

Figura 1 Distribuição percentual das UBS por regiões do Brasil: (A) horário fixo de funcionamento; e (B) e funcionamento no horário de almoço. Fonte: Banco de dados do PMAQ-AB Brasil, III ciclo 2017-2018 ${ }^{(18)}$. 
Em âmbito nacional, houve o predomínio de funcionamento de segunda a sexta-feira, em dois turnos e há pouca oferta de serviços em horários alternativos, incluindo os finais de semana. Destaca-se ainda que $99,3 \%$ das unidades abrem entre seis e oito horas e trinta minutos, e $89,4 \%$ encerram suas atividades entre dezesseis e dezoito horas e trinta minutos. Além disso, foram contabilizadas apenas 450 (1,6\%) unidades funcionando aos sábados e 196 (0,7\%) aos domingos, em todo o território nacional.

\section{Componente infraestrutura: Acessibilidade}

$\mathrm{Na}$ avaliação da acessibilidade às UBS brasileiras (Tabela 1), 87,1\% apresentaram todos os ambientes com sinalização, facilitando o acesso dos usuários; 77,3\% com entrada externa adaptada para cadeira de rodas, e 76,2\% com cadeira de rodas disponivel e em condição de uso, para deslocamento dos usuários.

Os aspectos mais comprometidos foram: existência de corrimão nos locais não nivelados $(19,6 \%)$ e o piso tátil para acesso às dependências da unidade (24,1\%). As UBS da região Norte apresentaram os piores indicadores referentes à acessibilidade.

Tabela 1 Distribuição do número de UBS, segundo presença ou não dos componentes de acessibilidade em suas dependências.

\begin{tabular}{|c|c|c|c|c|c|c|c|c|c|c|c|c|c|}
\hline \multirow{2}{*}{ Acessibilidade } & & \multicolumn{2}{|c|}{ Norte } & \multicolumn{2}{|c|}{ Nordeste } & \multicolumn{2}{|c|}{ Centro-Oeste } & \multicolumn{2}{|c|}{ Sudeste } & \multicolumn{2}{|c|}{ Sul } & \multicolumn{2}{|c|}{ Brasil } \\
\hline & & n & $\%$ & $\mathrm{n}$ & $\%$ & $\mathbf{n}$ & $\%$ & $\mathbf{n}$ & $\%$ & $\mathbf{n}$ & $\%$ & $\mathbf{n}$ & $\%$ \\
\hline Entrada externa adaptada & Sim & 1545 & 68,5 & 9239 & 76,7 & 1711 & 79,8 & 6491 & 77,9 & 3383 & 81,3 & 22369 & 77,3 \\
\hline para cadeira de rodas & Não & 710 & 31,5 & 2809 & 23,3 & 434 & 20,2 & 1840 & 22,1 & 777 & 18,7 & 6570 & 22,7 \\
\hline Corrimão nos locais não & Sim & 193 & 8,6 & 1991 & 16,5 & 312 & 14,5 & 1945 & 23,3 & 1241 & 29,8 & 5682 & 19,6 \\
\hline nivelados (ex.: escadas) & Não & 2062 & 91,4 & 10057 & 83,5 & 1833 & 85,5 & 6386 & 76,7 & 2919 & 70,2 & 23257 & 80,4 \\
\hline $\begin{array}{l}\text { Todas as portas internas } \\
\text { adaptadas para cadeira de }\end{array}$ & Sim & 1160 & 51,4 & 7051 & 58,5 & 1480 & 69,0 & 5398 & 64,8 & 3163 & 76,0 & 18252 & 63,1 \\
\hline rodas & Não & 1095 & 48,6 & 4997 & 41,5 & 665 & 31,0 & 2933 & 35,2 & 997 & 24,0 & 10687 & 36,9 \\
\hline Piso tátil para acesso as & Sim & 547 & 24,3 & 3240 & 26,9 & 599 & 27,9 & 1700 & 20,4 & 891 & 21,4 & 6977 & 24,1 \\
\hline dependências da unidade & Não & 1708 & 75,7 & 8808 & 73,1 & 1546 & 72,1 & 6631 & 79,6 & 3269 & 78,6 & 21962 & 75,9 \\
\hline $\begin{array}{l}\text { Todos os corredores } \\
\text { adaptados para cadeira de }\end{array}$ & Sim & 1134 & 50,3 & 7185 & 59,6 & 1550 & 72,3 & 5569 & 66,8 & 3233 & 77,7 & 18671 & 64,5 \\
\hline rodas & Não & 1121 & 49,7 & 4863 & 40,4 & 595 & 27,7 & 2762 & 33,2 & 927 & 22,3 & 10268 & 35,5 \\
\hline $\begin{array}{l}\text { Cadeira de rodas } \\
\text { disponivel e em condição }\end{array}$ & Sim & 1313 & 58,2 & 8645 & 71,8 & 1609 & 75,0 & 6840 & 82,1 & 3656 & 87,9 & 22063 & 76,2 \\
\hline de uso & Não & 942 & 41,8 & 3403 & 28,2 & 536 & 25,0 & 1491 & 17,9 & 504 & 12,1 & 6876 & 23,8 \\
\hline $\begin{array}{l}\text { Todos os ambientes } \\
\text { com sinalização (placa) }\end{array}$ & Sim & 1825 & 80,9 & 10567 & 87,7 & 1787 & 83,3 & 7288 & 87,5 & 3728 & 89,6 & 25195 & 87,1 \\
\hline facilitando o acesso & Não & 430 & 19,1 & 1481 & 12,3 & 358 & 16,7 & 1043 & 12,5 & 432 & 10,4 & 3744 & 12,9 \\
\hline & Sim & 115 & 5,1 & 262 & 2,2 & 49 & 2,3 & 138 & 1,7 & 47 & 1,1 & 611 & 2,1 \\
\hline & Não & 2140 & 94,9 & 11786 & 97,8 & 2096 & 97,7 & 8193 & 98,3 & 4113 & 98,9 & 28328 & 97,9 \\
\hline
\end{tabular}

Fonte: Banco de dados PMAQ-AB Brasil, III ciclo 2017-201818. 
Conforme mostra a Figura 2, a região Sul possui maior indice de sanitários para Pessoas com Deficiência (PcD) (68,2\%), e a região Norte o menor número (42,7\%).

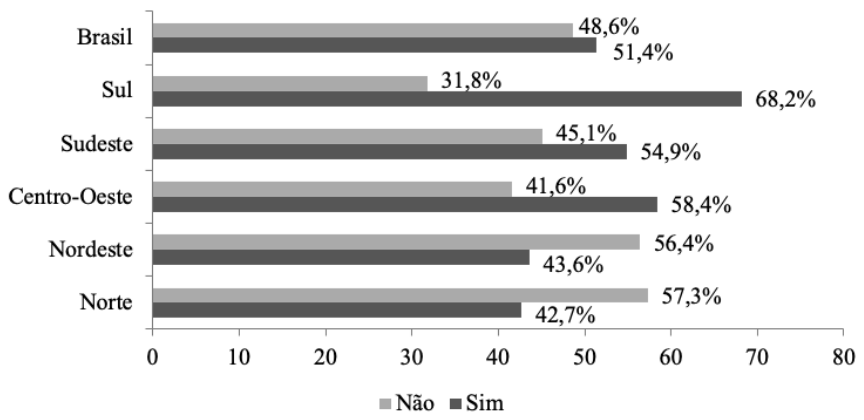

Figura 2 Distribuição do número de unidades de saúde, segundo a disponibilidade de sanitário com acessibilidade para PcD. Fonte: Banco de dados PMAQ-AB, III ciclo 2017-2018 ${ }^{18}$.

\section{Condições de Funcionamento}

No Brasil, 10,6\% das UBS estão instaladas em local provisório, 6,5\% estão em reforma e 4,6\% estão em ampliação, e $21,7 \%$ apresentam estrutura com padrões divergentes do estabelecido pelo Ministério da Saúde. A região Nordeste apresentou maior percentual de UBS em local provisório $(11,8 \%)$ e a região Norte, o maior percentual de UBS em reforma $(9,4 \%)$ e ampliação (7,1\%).

Tabela 2 Distribuição do número de unidades de saúde, segundo as condições de funcionamento.

\begin{tabular}{|c|c|c|c|c|c|c|c|c|c|c|c|c|c|}
\hline \multirow{2}{*}{\multicolumn{2}{|c|}{ Condições de Funcionamento }} & \multicolumn{2}{|c|}{ Norte } & \multicolumn{2}{|c|}{ Nordeste } & \multicolumn{2}{|c|}{ Centro-Oeste } & \multicolumn{2}{|c|}{ Sudeste } & \multicolumn{2}{|c|}{ Sul } & \multicolumn{2}{|c|}{ Brasil } \\
\hline & & $n$ & $\%$ & $n$ & $\%$ & $n$ & $\%$ & $n$ & $\%$ & $n$ & $\%$ & $n$ & $\%$ \\
\hline \multirow{2}{*}{ Em local provisório? } & Sim & 264 & 11,7 & 1426 & 11,8 & 230 & 10,7 & 855 & 10,3 & 296 & 7,1 & 3071 & 10,6 \\
\hline & Não & 1991 & 88,3 & 10622 & 88,2 & 1915 & 89,3 & 7476 & 89,7 & 3864 & 92,9 & 25868 & 89,4 \\
\hline \multirow{2}{*}{ Está em reforma? } & Sim & 212 & 9,4 & 769 & 6,4 & 142 & 6,6 & 523 & 6,3 & 242 & 5,8 & 1888 & 6,5 \\
\hline & Não & 2043 & 90,6 & 11279 & 93,6 & 2003 & 93,4 & 7808 & 93,7 & 3918 & 94,2 & 27051 & 93,5 \\
\hline \multirow{2}{*}{ Está em ampliação? } & Sim & 159 & 7,1 & 582 & 4,8 & 88 & 4,1 & 306 & 3,7 & 187 & 4,5 & 1322 & 4,6 \\
\hline & Não & 2096 & 92,9 & 11466 & 95,2 & 2057 & 95,9 & 8025 & 96,3 & 3973 & 95,5 & 27617 & 95,4 \\
\hline
\end{tabular}

Fonte: Banco de dados PMAQ-AB Brasil, III ciclo 2017-201818.

\section{DISCUSSÃO}

\section{Horário de Funcionamento}

Compreendendo o papel central da APS, como porta de entrada do SUS, destaca-se a importância do acesso da população a este nível de atenção. Entretanto, pesquisas indicam que o acesso às UBS não ocorre de maneira tão fácil e igualitária, e que muitos usuários são prejudicados pelo fato de as UBS funcionarem em horário comercial(12,15,19).

Dados do Instituto Brasileiro de Geografia e Estatística (IBGE) estimaram as horas de trabalho semanais exercidas por trabalhadores brasileiros, indicando que $72,7 \%$ atuam entre 40 e 49 horas ou mais. Comparados aos resultados deste estudo, esses dados sobre o horário de funcionamento possibilitam afirmar que os trabalhadores se encontram em situação desfavorável para o acesso aos serviços da $\mathrm{ABS}^{(20)}$.

Outro estudo trouxe como resultados que as características estruturais das UBS impactam no número de internações por Condições Sensiveis à Atenção Primária (CSAP), e suas taxas representam indicadores de acesso e qualidade da
APS $^{21}$. Assim, nas situações em que a ABS não é resolutiva, a demanda por internações hospitalares aumenta, criando despesas desnecessárias e evitáveis. Em relação a isso, as baixas coberturas em horários alternativos apontadas neste estudo são consideradas fator obstante para a população trabalhadora ter acesso as UBS(22).

Um estudo com dados do primeiro ciclo do PMAQ, apontou que $87,1 \%$ das UBS funcionavam em, pelo menos, dois turnos, nos cinco dias da semana(22). Já os resultados do terceiro ciclo demonstram um incremento no número de UBS atuando nestas condições (89,4\%). Quanto ao funcionamento durante o horário de almoço, no primeiro ciclo a cobertura era de $38,2 \%{ }^{(15)}$ e, no terceiro ciclo, o número cresceu para $46,3 \%$. Isso demonstra que, embora houvesse pouca diferença na evolução, o PMAQ instigou o aumento do acesso.

A PNAB orienta que as unidades funcionem por, pelo menos, 40 horas semanais durante, no mínimo, cinco dias da semana, nos 12 meses do ano. Os horários alternativos dependem das pactuações das instâncias de participação social, 
"desde que atendam expressamente a necessidade da população"(16). Logo, a maioria das UBS do Brasil atuam de acordo com o horário mínimo estabelecido pela PNAB, no entanto, não atendem expressamente as demandas populares, como no caso da situação dos trabalhadores, que não têm disponibilidade de atendimento fora do horário de trabalho. Todavia, destaca-se que, qualquer modalidade adotada para solucionar esta problemática deve considerar os princípios e diretrizes do SUS, no âmbito da APS.

\section{Acessibilidade e Condições de Funcionamento das UBS}

Segundo a Associação Brasileira de Normas Técnicas (ABNT), para que um espaço ou edificação seja considerado acessivel, este deve ser utilizado por qualquer pessoa, o que inclui pessoas com mobilidade reduzida(23). Destaca-se também que as condições de acesso pela PcD estão diretamente relacionadas à acessibilidade, que pode ser compreendida como a possibilidade e condição de uso com segurança e autonomia.

A pesquisa de Albuquerque e colaboradores apontou que, em 97\% das UBS de Pernambuco não existia banheiros adaptados e 98\% não apresentava corrimão ${ }^{(24)}$. Outra pesquisa indicou que poucos estados brasileiros apresentam UBS com coberturas potenciais de acessibilidade para PcD superiores a $70 \%$, evidenciando baixo desempenho em estados da região Centro-Oeste e melhor desempenho nos estados da região Nordeste ${ }^{15}$. Considerando os resultados obtidos neste estudo, nota-se que, ao longo dos anos, a acessibilidade não apresentou avanços significativos nos serviços de APS.

Ressalta-se, ainda, que a utilização de imóveis com características domiciliares demonstra a pouca valorização que se dá para os aspectos estruturais das unidades da APS. É importante que a concepção arquitetônica das UBS tenha uma boa ambiência, se integre ao entorno, de acordo com os valores da comunidade local, e que o acesso seja facilitado. Para isso, sua estrutura deve ser implantada de acordo com o preconizado pelo Manual de Estrutura Física das UBS, do Ministério da Saúde ${ }^{(25)}$, em conformidade com a PNAB ${ }^{(16)}$.

Considerando que o cenário relativo às condições de funcionamento das UBS, evidenciado neste estudo, contraria as leis e normas estabelecidas pelo Ministério da Saúde, e a melhora da acessibilidade nas UBS para PcD, idosos e demais usuários, exige que a entrada externa, as portas internas e os corredores sejam adaptados, para estarem disponiveis e em condições de uso, facilitando o acesso de todos.

\section{Desigualdades regionais}

Os resultados deste estudo representam um alerta quan- to à situação da estrutura das UBS, sobretudo por apontar regiões com baixos indicadores de acesso e acessibilidade. Dados do Ministério da Saúde apontam que, em setembro de 2019, a região Nordeste apresentou melhor cobertura (84,4\%), seguida pelas regiões Sul (79,2\%), Norte (71,1\%), Centro-Oeste $(71,0 \%)$ e Sudeste $(68,2 \%)^{(26)}$. Já em relação à saúde suplementar, compreendida como as atividades que envolvem planos e seguros de saúde, a região Norte apresentou menor cobertura, com 1.729 .041 beneficiários e a região Sudeste, a maior cobertura, com 28.658.032 beneficiários na saúde suplementar ${ }^{(27)}$.

Tais dados tornam-se relevantes, pois as UBS da Região Norte foram as que mais apresentaram fragilidades nos critérios analisados neste estudo, tendo a terceira menor cobertura da APS do país e também a menor cobertura nacional na modalidade saúde suplementar. Esses resultados evidenciam o comprometimento na capacidade de alcance da população que reside nessa região aos serviços de saúde, e a menor qualidade nos critérios referentes ao acesso e à acessibilidade aos serviços de APS.

\section{Limitações do Estudo}

Aponta-se uma limitação referente aos dados sobre os horários de funcionamento das UBS que, da forma como foram registrados não permitiu estabelecer o horário exato de funcionamento diário das unidades, sendo possivel identificar apenas em quais turnos as UBS funcionavam. Isto decorreu da dificuldade em mesclar o horário de início e encerramento dos serviços com as UBS que atuavam durante o horário do almoço.

\section{Contribuições para a Prática}

O estudo contribui com a produção de conhecimentos importantes para a gestão da APS no Brasil, a partir da caracterização do acesso e da acessibilidade na estrutura das UBS, estratificadas de acordo com a adesão ao PMAO, em cada região do Brasil. Os resultados corroboram discussões e tomada de decisões dos gestores de saúde nos diferentes estados e regiões do país.

\section{CONCLUSÃO}

Houve disparidades regionais no acesso e na acessibilidade às Unidades Básicas de Saúde brasileiras. Na avaliação geral, o componente organizacional aponta conformidade com o preconizado pela PNAB. Já no componente infraestrutura, a acessibilidade indicou baixos percentuais, evidenciando comprometimento no acesso dos usuários, o que demanda maiores investimentos públicos.

Frente ao exposto, é relevante implementar novos mé- 
todos de monitoramento local, de forma a aprimorar e direcionar ações de melhoria de acesso e acessibilidade da população, ressaltando a importância da estrutura, cuja inadequação pode influenciar o processo de trabalho e a qualidade da assistência na APS.

Para a prática gerencial, a realização de avaliação da estrutura é um instrumento importante e deve ser estimulada, aplicada e discutida. Portanto, almeja-se que este estudo possa contribuir de forma significativa para o empoderamento de decisões, nos diferentes níveis da gestão da APS no SUS.
CONTRIBUIÇÃO DOS AUTORES: ECCP: Trabalhou na redação, análise e interpretação de dados. TANCB: Trabalhou na redação, análise e interpretação de dados. ML: Trabalhou na análise e interpretação de dados. GRONF: Trabalhou na análise, interpretação dos dados, na revisão crítica e aprovação da versão final a ser publicada. LGL: Trabalhou na revisão crítica e aprovação da versão final a ser publicada. HHCP: Trabalhou na análise, interpretação dos dados. EPB: Trabalhou na revisão crítica e aprovação da versão final a ser publicada. CLFC: Trabalhou no desenho do estudo, na revisão crítica e aprovação da versão final a ser publicada.

\section{REFERÊNCIAS}

1. Bousquat A, Giovanella L, Fausto MCR, Fusaro ER, Mendonça MHM, Gagno J et al. Tipologia das unidades básicas de saúde brasileiras. Tipologia da estrutura das unidades básicas de saúde brasileiras: os 5 R. Cad. Saúde Pública [Internet]. 2017 [cited 2020 Mar 23]; 33(8):e00037316. Available from: https://doi.org/10.1590/0102-311×00037316.

2. Moreira KS, Lima CA, Vieira MA, Costa SM. Avaliação da Infraestrutura das Unidades de Saúde da Família e Equipamentos para ações na Atenção Básica. Cogitare Enferm. [Internet]. 2017 [cited 2020 Mar 23]; (22)2:e51283. Available from: http://dx.doi.org/10.5380/ce.v22i2.51283.

3. Neves GR, Duro SMS, Muñiz J, Castro TRP, Facchini LA, Tomasi E. Estrutura das unidades básicas de saúde para atenção às pessoas com diabetes: Ciclos I e II do Programa Nacional de Melhoria do Acesso e da Qualidade. Cad. Saúde Pública [Internet]. 2018 [cited 2020 Mar 23]; 34(4):e00072317. Available from: https://doi.org/10.1590/0102-311x00072317.

4. Moura BLA, Cunha RC, Fonseca ACF, Aquino A, Medina MG, Vilasboas ALQ et al. Atenção primária à saúde: estrutura das unidades como componente da atenção à saúde. Rev. Bras. Saude Mater. Infant. [Internet]. 2010 [cited 2020 Mar 23]; 10(Suppl 1):s69-s81. Available from: https://doi.org/10.1590/S1519-38292010000500007.

5. Leite TRC, Lopes MSV, Maia ER, Cavalcante EGR. Avaliação da estrutura da atenção primária à saúde na atenção à hanseníase. Enferm. Foco [Internet]. 2019 [cited 2020 Mar 23]; 10(4):73-8. Available from: http://revista.cofen.gov.br/index.php/enfermagem/article/view/2216/608.

6. Donabedian A. The Quality of Care: How Can It Be Assessed? JAMA. [Internet]. 1988 [cited 2020 Mar 23]:260(12):1743-

8. Available from: https://jamanetwork.com/journals/jama/article-abstract/374139.

7. Ministério da Saúde (BR). Portaria 1.645, de 02 de outubro de 2015. Dispõe sobre o Programa Nacional de Melhoria do Acesso e da Qualidade da Atenção Básica (PMAQ-AB). Diário Oficial da União 5 out. 2015; Seção 1, p. 668. [cited 2020 Mar 23]. Available from: https:// bvsms.saude.gov.br/bvs/saudelegis/gm/2015/prt1645_01_10_2015.html.

8. Amaral FLJS, Motta MHA, Silva LPG, Alves SB. Fatores associados com a dificuldade no acesso de idosos com deficiência aos serviços de saúde. Ciênc. saúde coletiva [Internet]. 2012 [cited 2020 Mar 23]; 17(11):2991-3001. Available from: https://doi.org/10.1590/S141381232012001100016 .

9. Cunha ABO, Silva LMV. Acessibilidade aos serviços de saúde em um município do Estado da Bahia, Brasil, em gestão plena do sistema. Cad. Saúde Pública [Internet]. 2010 Apr [cited 2020 Mar 23]; 26(4):725-37. Available from: https://doi.org/10.1590/S0102-311X2010000400015.

10. Siqueira FCV, Facchini LA, Silveira DS, Piccini RX, Thumé E, Tomasi E. Barreiras arquitetônicas a idosos e portadores de deficiência física: um estudo epidemiológico da estrutura física das unidades básicas de saúde em sete estados do Brasil. Ciênc. saúde coletiva [Internet]. 2009 [cited 2020 Mar 23]; 14(1):39-44. Available from: https://doi.org/10.1590/S1413-81232009000100009.

11. Janssen M. Fonseca SC. Alexandre GC. Avaliação da dimensão estrutura no atendimento ao pré-natal na Estratégia Saúde da Familia: potencialidades do PMAQ-AB. Saúde debate [Internet]. 2016 [cited 2020 Mar 23]; 40(111):140-52. Available from: https://doi. org/10.1590/0103-1104201611111. 
12. Pessoa BHS, Gouveia EAH, Correia IB. Funcionamento 24 horas para Unidades de Saúde da Familia: uma solução para ampliação de acesso? Um ensaio sobre as "Upinhas" do Recife. Rev Bras Med Fam Comunidade [Internet]. 2017 [cited 2020 Mar 23]:12(39):1-9. Available from: https://doi.org/10.5712/rbmfc12(39)1529.

13. Starfield B. Atenção primária: equilibrio entre necessidades de saúde, serviços e tecnologia. Brasilia (DF): UNESCO, Ministério da Saúde; 2002 [cited 2020 Apr 16]. Available from: https://bvsms.saude.gov.br/bvs/publicacoes/atencao_primaria_pl.pdf.

14. Fekete MC. Estudo da acessibilidade na avaliação dos serviços de saúde. In: Santana JP, Santos I, Fekete MC, Galvão EA, Mandelli MJ, Penna MLF, et al., organizadores. Desenvolvimento gerencial de Unidades Básicas do Sistema Único de Saúde (SUS). Brasilia (DF): Organização Pan-Americana da Saúde; 1997. p. 177-84.

15. Poças KC, Freitas LRS, Duarte EC. Censo de estrutura da Atenção Primária à Saúde no Brasil (2012): estimativas de coberturas potenciais. Epidemiol. Serv. Saúde [Internet]. 2017 [cited 2020 Mar 23]; 26(2):275-84. Available from: https://doi.org/10.5123/s1679-49742017000200005. 16. Ministério da Saúde (BR). Portaria 2.436/GM, de 21 de setembro de 2017. Aprova a Política Nacional de Atenção Básica, estabelecendo a revisão de diretrizes para a organização da Atenção Básica, no âmbito do Sistema Único de Saúde (SUS). Diário Oficial da União 22 set. 2017 ; Seção 1. p. 68. [cited 2020 Mar 23]. Available from: https://bvsms.saude.gov.br/bvs/saudelegis/gm/2017/prt2436_22_09_2017.html.

17. Cunha CLF, Ramalho NM. Protocolos de enfermagem: promovendo o acesso e qualidade da assistência na Atenção Primária à Saúde. Enferm. Foco [Internet]. 2019 [cited 2020 Mar 23]; 10(4):6. Available from: http://revista.cofen.gov.br/index.php/enfermagem/article/ download/3209/597.

18. Ministério da Saúde (BR). Secretaria de Atenção Primária à Saúde. Programa de Melhoria da Atenção Básica - 3 @ Ciclo [cited 2020 Abr 16] Available from: https://aps.saude.gov.br/ape/pmaq/ciclo3/.

19. Quintero MCF, Vendramini SHF, Santos MLSG, Santos MR, Gazetta CE, Lourenção LG et al. Acesso ao diagnóstico da tuberculose em municipio brasileiro de médio porte. Rev. salud pública [Internet]. 2018 [cited 2020 Mar 23]; 20(1):103-9. Available from: https://doi. org/10.15446/rsap.V20n1.64177.

20.Instituto Brasileiro de Geografia e Estatistica (IBGE) (BR)Brasil em Sintese. Trabalho. 2012. [cited 2020 Mar 23]. Available from: https:// brasilemsintese.ibge.gov.br/trabalho.

21. Nedel FB, Facchini LA, Martin M, Navarro A. Características da atenção básica associadas ao risco de internar por condições sensíveis à atenção primária: revisão sistemática da literatura. Epidemiol. Serv. Saúde [Internet]. 2010 [cited 2020 Mar 23]; 19(1):61-75. Available from: http://dx.doi.org/10.5123/S1679-49742010000100008.

22. Araujo WRM, Queiroz RCS, Rocha TAH, Silva NC, Thumé E, Tomasi E et al. Estrutura e processo de trabalho na atenção primária e internações por condições sensíveis. Rev. Saúde Pública [Internet]. 2017 [cited 2020 Mar 23]; 51:75. Available from: https://doi.org/10.11606/ S1518-8787.2017051007033.

23. Associação Brasileira de Normas Técnicas (ABNT). NBR 9050: Acessibilidade a edificações, mobiliário, espaços e equipamentos urbanos. Rio de Janeiro; 2004. [cited 2020 Mar 23]. Available from: http://www.mpf.mp.br/atuacao-tematica/pfdc/institucional/grupos-de-trabalho/ inclusao-pessoas-deficiencia/atuacao/legislacao/docs/norma-abnt-NBR-9050.pdf/view.

24. Albuquerque MSV, Lyra TM, Farias SF, Mendes MFM, Martelli PJL. Acessibilidade aos serviços de saúde: uma análise a partir da Atenção Básica em Pernambuco. Saúde debate [Internet]. 2014 [cited 2020 Mar 23]; 38(spe):182-94. Available from: https://doi.org/10.5935/01031104.20145014

25. Ministério da Saúde (BR). Secretaria de Atenção à Saúde. Departamento de Atenção Básica. Manual de estrutura física das Unidades Básicas de Saúde: Saúde da Familia. 2 ed. Brasilia; 2008. [cited 2020 Mar 23]. Available from: https://bvsms.saude.gov.br/bvs/publicacoes/ manual_estrutura_ubs.pdf.

26. Ministério da Saúde(BR). Secretaria de Atenção Primária à Saúde. Departamento de Saúde da Família. Cobertura da Atenção Básica: setembro de 2019 [cited 2020 Mar 23]. Available from: https://egestorab.saude.gov.br/paginas/acessoPublico/relatorios/relHistoricoCoberturaAB. xhtml.

27. Agência Nacional de Saúde Suplementar (ANS). Sistema de Informações de Beneficiários (SIB). Cobertura planos de saúde por regiões: setembro de 2019. [cited 2020 Mar 23]. Available from: http://www.ans.gov.br/anstabnet/cgi-bin/tabnet?dados/tabnet_br.def. 